\title{
Investigating the molecular mechanism of Compound Danshen Dropping Pills for the treatment of epilepsy by utilizing network pharmacology and molecular docking technology
}

\author{
Dan Huang ${ }^{1 \#}$, Xiaolong Wen ${ }^{2 \#}$, Chuansen Lu ${ }^{1}$, Bo Zhang ${ }^{1}$, Zongjun Fu ${ }^{1}$, Yingliu Huang ${ }^{1}$, Kun Niu ${ }^{3}$, \\ Fan Yang ${ }^{3}$
}

${ }^{1}$ Department of Neurology, Hainan General Hospital, Hainan Affiliated Hospital of Hainan Medical University, Haikou, China; ${ }^{2}$ Department of Pharmacy, The Second Affiliated Hospital of Hainan Medical University, Haikou, China; ${ }^{3}$ College of Traditional Chinese Medicine, Hainan Medical University, Haikou, China

Contributions: (I) Conception and design: F Yang, K Niu, D Huang, Y Huang; (II) Administrative support: X Wen, K Niu; (III) Provision of study materials: C Lu, B Zhang, Z Fu, K Niu; (IV) Collection and assembly of data: D Huang, X Wen; (V) Data analysis and interpretation: F Yang, K Niu; (VI) Manuscript writing: All authors; (VII) Final approval of manuscript: All authors.

\#These authors contributed equally to this work.

Correspondence to: Fan Yang; Kun Niu. College of Traditional Chinese Medicine, Hainan Medical University, No. 3 Xueyuan Road, Haikou 571199, China. Email: tcmyang@hainmc.edu.cn or yangfanhmu@163.com; ikunniu@hainmc.edu.cn.

Background: Compound Danshen Dropping Pills (CDDP) is widely used in clinical treatment of epilepsy. But the underlying active ingredients and molecular mechanisms are unclear. Our study aims to investigate the active components and functional mechanisms of CDDP in treating epilepsy using a network pharmacology approach.

Methods: Candidate constituents and targets of CDDP were searched on the Traditional Chinese Medicine Systems Pharmacology database. NCBI and Genecards were used to establish a database of epilepsy targets. Next, used Cytoscape software, the interactive network diagram of "drug-active component-target" was drawn. Based on the STRING database we constructed protein-protein interaction network and analyzed protein-protein interaction relationships. Gene ontology analysis and Kyoto Encyclopedia of Genes and Genomes pathway enrichment analysis were performed for the common targets. Molecular docking provided an evaluation tool for verifying the combination of components and targets, which was performed using Auto-dock.

Results: Sixty bioactive components, corresponding to 79 therapeutic targets for epilepsy, were successfully identified. Functional enrichment analysis showed that CDDP plays a pharmacological role in the treatment of epilepsy by regulating serotonergic synapses, morphine addiction, nicotine addiction and other pathways, as well as the $N F-\kappa B$ signaling pathway. Molecular docking analysis showed that representative components may be closely bound to key targets.

Conclusions: This network pharmacology study revealed the synergistic effects of multiple components, targets, and pathways of CDDP in the treatment of epilepsy, which will deepen our understanding of the underlying molecular mechanisms of CDDP in the treatment of epilepsy and lay the foundation for further experimental studies.

Keywords: Compound Danshen Dropping Pills (CDDP); epilepsy; molecular docking; network pharmacology

Submitted Nov 25, 2021. Accepted for publication Feb 22, 2022.

doi: $10.21037 / \mathrm{atm}-22-195$

View this article at: https://dx.doi.org/10.21037/atm-22-195 


\section{Introduction}

Epilepsy is characterized by spontaneous convulsive behavior and abnormal brain discharge, and it is a very common chronic nervous system disease. Its incidence range is broad, and approximately $0.5-1 \%$ of people suffer from epilepsy worldwide (1). There are about 70 million epilepsy patients in the world (2).

The pathogenesis of epilepsy is complex, including abnormal excitation of the central nervous system, abnormal discharge of ion channels, genetic factors, and immune system diseases (3). It is considered that the enhancement of cortical excitability caused by the imbalance of brain excitation and inhibition mechanism are vital for pathophysiological of epilepsy (4). Epidemiological investigation has shown that the prevalence of epilepsy globally is close to $1 \%$, and the total number of epilepsy patients in the world has exceeded 50 million (5). Furthermore, epilepsy in the elderly may significantly increase the risk of fracture or abnormal fall injury (6-8). At the same time, epilepsy patients are more prone to sudden death of unknown cause (9). At present, the treatment of epilepsy is mainly pharmacological, which can effectively control seizures, but also increase the risk of suicide or other various concurrent diseases to a certain extent, such as exfoliative dermatitis, polycystic ovary syndrome, etc. (10-12). Therefore, epilepsy has become one of the major diseases endangering human health.

At present, antiepileptic drugs such as sodium valproate, carbamazepine, and levetiracetam are mainly used to treat the disease. However, there are disadvantages including poor curative effect, easy tolerance, as well as significant toxicity and side effects. Traditional Chinese medicine can make up for these defects and play an important role in the treatment of epilepsy; it has good curative effect, with long-lasting efficacy and few side effects. Compound Danshen Dropping Pills (CDDP) can reduce inflammatory reactions, control seizures, improve learning and memory function, and repair hippocampal neuron damage. The main components of CDDP are borneol, Salvia miltiorrhiza and Panax notoginseng. It is a traditional Chinese medicine dropping pill developed based on compound Danshen tablets under the guidance of traditional Chinese medicine theory. It has a wide range of clinical applications, such as the treatment of coronary heart disease, acute myocardial infarction and other diseases. In addition, it is widely used in clinical treatment of epilepsy, but its pharmacological mechanism is still unclear (3).
Network pharmacology emphasizes the strategy shift from "one target, one drug" strategy to the new method of "network target, multi-component" (13-16). CDDP and other traditional Chinese medicines have the potential to promote the development of multi-component and multitarget synergistic therapy for epilepsy.

With network pharmacology, we investigated the underlying anti-epileptic mechanism of CDDP. Firstly, we obtained the potential target genes of CDDP, and then studied the pathways shared by CDDP and the molecular targets of epilepsy. We conducted molecule docking to investigate the interaction between important compounds and targets. Compared with previous studies, we calculated the Root Mean Square Deviation (RMSD) value of docking to ensure the accuracy of docking results. Our results may help to clarify how CDDP can effectively treat epilepsy and promote the developing new drugs.

We present the following article in accordance with the STREGA reporting checklist (available at https://atm. amegroups.com/article/view/10.21037/atm-22-195/rc).

\section{Methods}

\section{Target genes related to epilepsy}

Epilepsy-related genes were searched through the National Center for Biotechnology Information (https://www.ncbi. nlm.nih.gov/) (17) and Genecards (https://www.genecards. org/) (18) databases using "epilepsy" and "Homo sapiens" as the search terms. The targets of both databases were synthesized to delete duplicate values and obtain the relevant epilepsy targets. Seven hundred and twentynine targets were obtained from the NCBI, and 2,088 targets were retrieved with Genecards (score $\geq 1$ ). After excluding the duplicated targets, we obtained a total of 2,234 targets. The study was conducted in accordance with the Declaration of Helsinki (as revised in 2013).

\section{Prediction of protein-encoding genes that are targeted by CDDP}

The Traditional Chinese Medicine Systems Pharmacology Database and Analysis Platform (TCMSP) is a frequently used database to study mechanisms of Traditional Chinese medicines (19). We searched the TCMSP for the components of CDDP, including Salvia miltiorrhiza Bge., Panax notoginseng (Burk.) F. H. Chen, Borneol. Each component was obtained by using oral bioavailability 
(OB) $\geq 30 \%$ and drug likeness (DL) $\geq 0.18$ as the screening parameters (20).

\section{Potential target genes of CDDP treating epilepsy}

Shared target genes between epilepsy and the CDDPrelated genes were intersected using a Venn diagram. We considered shared genes to be potential target genes of CDDP treating epilepsy.

\section{The "drug-active component-target" network construction}

CDDP active components and common genes were imported into the cytoscape 3.8.1. to build a "drug-active ingredient-target" network.

\section{Construction of the target gene network}

With STRING database (http://string-db.org/cgi/input.pl) and defining the species as "Homo sapiens" (21), a proteinprotein interaction (PPI) network of intersecting genes of CDDP acting on epilepsy was constructed. The PPI data was downloaded and saved in TSV format. In addition, Cytoscape software (Institute of Systems Biology, the US) and its network analyzer tools were used to determine the accuracy of each target gene when selecting the core target.

\section{Cluster analysis}

Using Molecular Complex Detection (MCODE), the most important modules of PPI network were identified (22).

\section{Analysis of gene ontology (GO) and Kyoto Encyclopedia of Genes and Genomes (KEGG) pathway enrichment}

Cytoscape clue GO was used for GO analysis, KEGG analysis of final target. The GO and KEGG pathway analyses were set as $\mathrm{P}<0.05$.

\section{Molecular docking}

Molecular docking was intended to validate interactions between the central targets and major active ingredients. We downloaded the protein crystal structure by searching the Research of Cooperative Organization for structural bioinformatics (RCSB) protein database (http://www.pdb. org/). The conformation of the protein was modified using Pymol (Schrödinger, the US) and Autodock1.5.6 (Olson
Laboratory of Scripps Institute, the US) software, including the removal of ligand and water, the addition of hydrogen, the optimization of amino acids, and the calculation of charge. Structural files of the key components are then downloaded through TCMSP, and with Chem3D software (Cambridgesoft, the US), their energy is minimized. The natural format of components and proteins was converted to the PDBQT format. Using AutoDock Vina, we performed molecular docking, and Discovery Studio 2019 (Beijing Chuangteng Technology Co., Ltd., China) was used to visualize docking results.

\section{Results}

\section{Main components of CDDP}

We obtained 336 chemical components of CDDP through TCMSP, including 76 components with $\mathrm{OB} \geq 30 \%$ and $\mathrm{DL} \geq 0.18$. 65 components were included in Radix Salviae (Danshen), three components were included Borneolum Syntheticum (Bingpian), and eight compounds were included Panax Notoginseng (Burk.) F. H. Chen Ex C. Chow (Sanqi). After eliminating compounds without targets, 60 compounds remained. Table 1 and Figure $1 A$ had shown the active components and their $\mathrm{OB}$ and DL values.

\section{The targets of CDDP in the treatment of epilepsy}

Through TCMSP database, we searched 197 CDDP targets. According to the NCBI and Genecards database, 2,234 epilepsy targets were obtained. Finally, 79 CDDP targets were identified in epilepsy treatment (Figure 1B).

\section{'Drug-active component-target' network analysis}

We used cytoscape software to construct "drug- ingredienttarget" networks. A total of 336 CDDP components were identified from the TCMSP. In this study, 76 bioactive components were screened, 16 of which had no target. Through TCMSP database, 60 components were obtained. The red nodes represented the drugs of CDDP, and the green nodes represented the CDDP active ingredients. Blue nodes represented potential CDDP targets, and the edges indicate the association between ingredients and targets (as shown in Figure 2).

\section{PPI network analysis}

Using Cytoscape 3.8.1 and based on 79 candidate targets, 
Table 1 Basic information of CDDP components

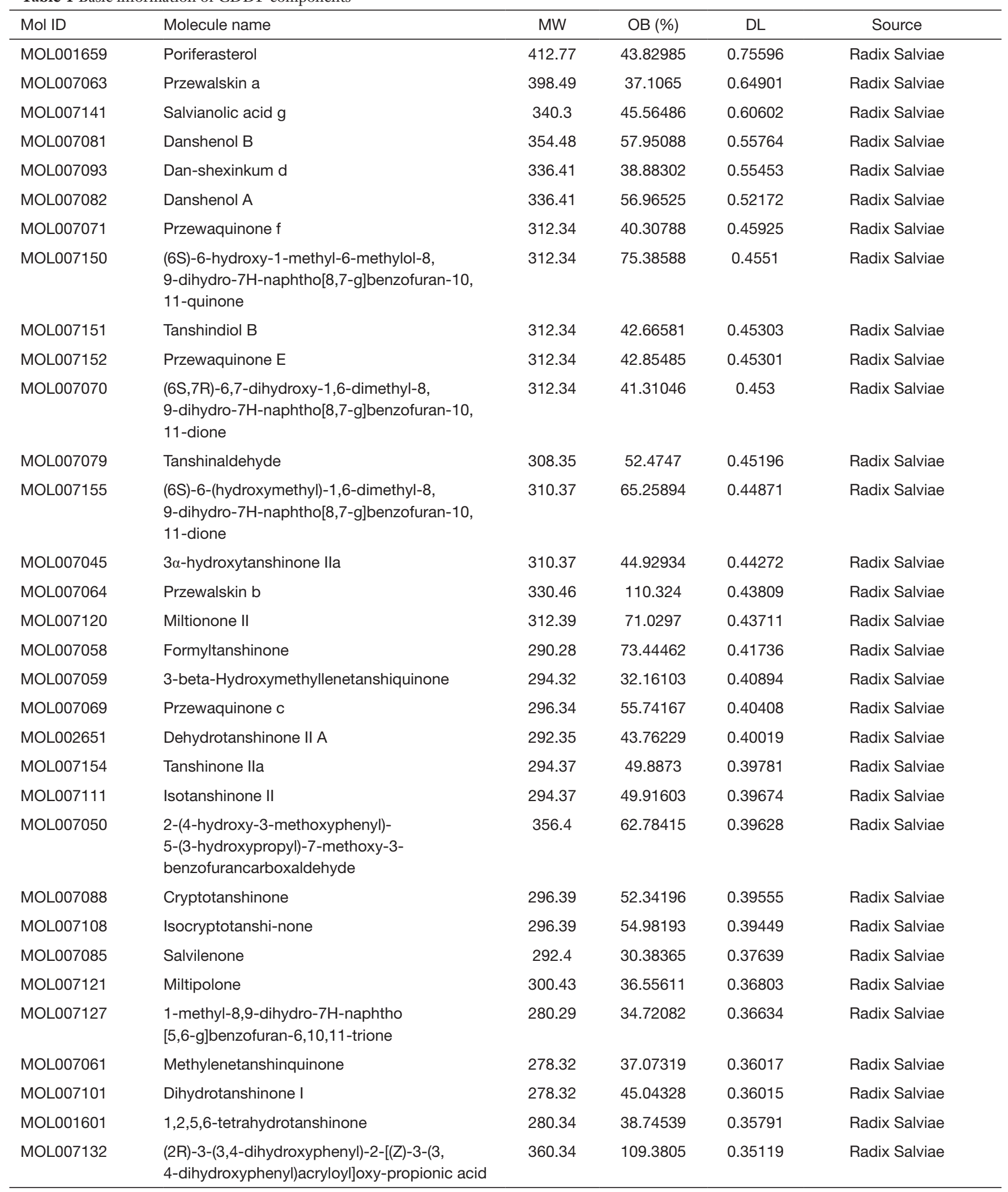

Table 1 (continued) 
Table 1 (continued)

\begin{tabular}{|c|c|c|c|c|c|}
\hline Mol ID & Molecule name & MW & $\mathrm{OB}(\%)$ & $\mathrm{DL}$ & Source \\
\hline MOL007125 & Neocryptotanshinone & 314.41 & 52.488 & 0.32306 & Radix Salviae \\
\hline MOL007100 & Dihydrotanshinlactone & 266.31 & 38.68477 & 0.32227 & Radix Salviae \\
\hline MOL007048 & $\begin{array}{l}\text { (E)-3-[2-(3,4-dihydroxyphenyl)-7-hydroxy- } \\
\text { benzofuran-4-yl]acrylic acid }\end{array}$ & 312.29 & 48.24363 & 0.31229 & Radix Salviae \\
\hline MOL007094 & Dan shen spiroketal lactone & 282.36 & 50.43128 & 0.3067 & Radix Salviae \\
\hline MOL007105 & Epidanshen spiroketal lactone & 284.38 & 68.27316 & 0.30549 & Radix Salviae \\
\hline MOL007156 & Tanshinone VI & 296.34 & 45.63731 & 0.29549 & Radix Salviae \\
\hline MOL007036 & $\begin{array}{l}\text { 5,6-dihydroxy-7-isopropyl-1,1-dimethyl-2,3- } \\
\text { dihydrophenanthren-4-one }\end{array}$ & 298.41 & 33.76525 & 0.28585 & Radix Salviae \\
\hline MOL000569 & Digallate & 322.24 & 61.84862 & 0.25635 & Radix Salviae \\
\hline MOL007122 & Miltirone & 282.41 & 38.75699 & 0.25418 & Radix Salviae \\
\hline MOL007107 & C09092 & 286.5 & 36.06949 & 0.2474 & Radix Salviae \\
\hline MOL000006 & Luteolin & 286.25 & 36.16263 & 0.24552 & Radix Salviae \\
\hline MOL007145 & Salviolone & 268.38 & 31.72415 & 0.23568 & Radix Salviae \\
\hline MOL007124 & Neocryptotanshinone ii & 270.35 & 39.46299 & 0.23157 & Radix Salviae \\
\hline MOL007041 & 2-isopropyl-8-methylphenanthrene-3,4-dione & 264.34 & 40.86015 & 0.22897 & Radix Salviae \\
\hline MOL007143 & Salvilenone I & 270.4 & 32.43471 & 0.22895 & Radix Salviae \\
\hline MOL005344 & ginsenoside rh2 & 622.98 & 36.31951 & 0.55868 & $\begin{array}{l}\text { Panax Notoginseng (Burk.) } \\
\text { F. H. Chen Ex C. Chow }\end{array}$ \\
\hline MOL000098 & Quercetin & 302.25 & 46.43335 & 0.27525 & $\begin{array}{l}\text { Panax Notoginseng (Burk.) } \\
\text { F. H. Chen Ex C. Chow }\end{array}$ \\
\hline MOL001494 & Mandenol & 308.56 & 41.9962 & 0.19321 & $\begin{array}{l}\text { Panax Notoginseng (Burk.) } \\
\text { F. H. Chen Ex C. Chow }\end{array}$ \\
\hline MOL001792 & DFV & 256.27 & 32.76272 & 0.18316 & $\begin{array}{l}\text { Panax Notoginseng (Burk.) } \\
\text { F. H. Chen Ex C. Chow }\end{array}$ \\
\hline
\end{tabular}

CDDP, Compound Danshen Dropping Pills; WM, molecular weight; OB, oral bioavailability; DL, drug likeness. 
A

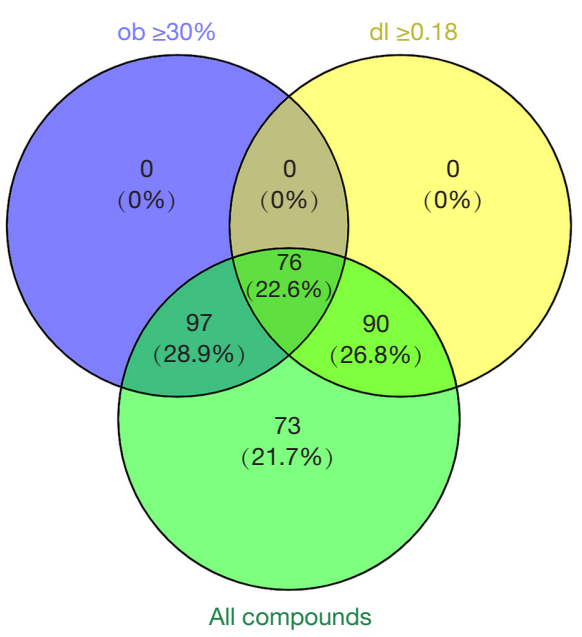

B
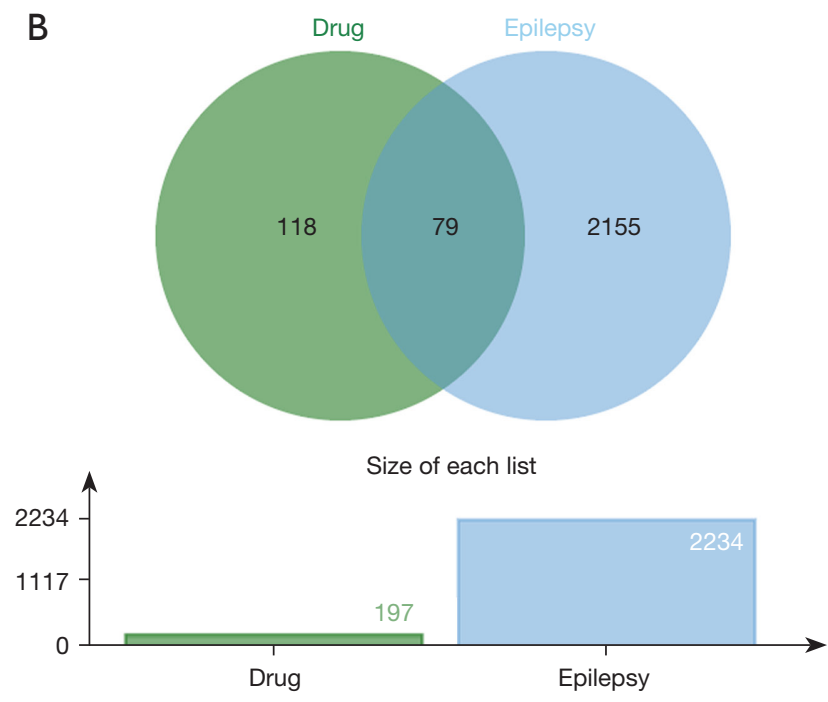

Number of elements : specific (1) or shared by $2,3, \ldots$ lists

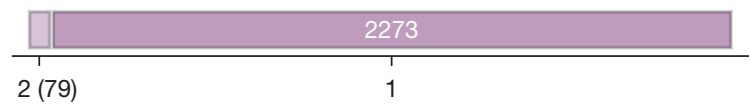

Figure 1 Compound Danshen Dropping Pills (CDDP) screening of bioactive compounds. (A) Venn diagram: based on absorption, distribution, metabolism, excretion (ADME)-related models, 76 bioactive components were screened [blue section stands for the components of oral bioavailability $(\mathrm{OB}) \geq 30 \%$, yellow section stands for drug likeness (DL) $\geq 0.18$; (B) Venn diagram of CDDP- and epilepsy-related targets; 79 common targets were obtained.

we established a PPI network. There were 79 nodes and 984 edges, where the average node degree was 24.9 and the local clustering coefficient was 0.698 (Figure 3). MCODE network analysis showed five clusters (Figure $4 A-4 E$ ), and the scores were $31.737,3.333,3,3$, and 3 respectively. These proteins have important function in the PPI network, and the top five proteins were $A K T$ serine/threonine kinase 1 (AKT1), cellular tumor antigen p53 (TP53), interleukin-6 (IL-6), tumor necrosis factor (TNF) (Figure 5A). The 11 compounds with the most targets are shown in Figure $5 B$.

\section{Analysis of GO function and KEGG patbway enrichment}

Through GO enrichment analysis, we obtained a total of 4,270 GO items, including 4,145 biological processes (BP) items, 11 cell components (CC) items, and 115 molecular functions (MF) items. The results showed that CDDP treatment of epilepsy BP was mainly related to the activation of adenylate cyclase activity, odontogenesis of dentincontaining tooth, including dentin, androgen metabolism, response to toxic substances, response to estrogen, and cellular response to estrogen stimulus. CC was mainly involved in platelet alpha particles cavity, projection of cytoplasm, and dendrites neuronal cytoplasm, etc. MF was mainly involved in the activation of protein kinase B activity, tau protein kinase activity, protein kinase $\mathrm{C}$ binding, and adenylate cyclase activity, as shown in Figure $6 \mathrm{~A}$.

Based on KEGG pathway enrichment analysis, a total of 103 pathways were obtained. According to the $\mathrm{P}$ value, the top 20 items were shown in Figure $6 B$. These included the serotonergic synapse, morphine addiction, nicotine addiction, $N F-\kappa B$, and Nod-like receptor signaling pathways.

\section{Molecular docking analysis}

Quercetin, luteolin, and tanshinone IIa were selected as the key active ingredients to perform molecular docking with AKT1, IL6, and TP53 (the basic information is shown in Table 2). The key active ingredients and hub targets were verified by molecular docking using the binding energy $\leq-5.0 \mathrm{kcal} / \mathrm{mol}$ as the standard (18). Docking results (shown in Figures 7-9 and Table 3) showed that the binding free energy of the key active ingredients of epilepsy and the hub target was far less than $-5.0 \mathrm{kcal} / \mathrm{mol}$, and the RMSD was 


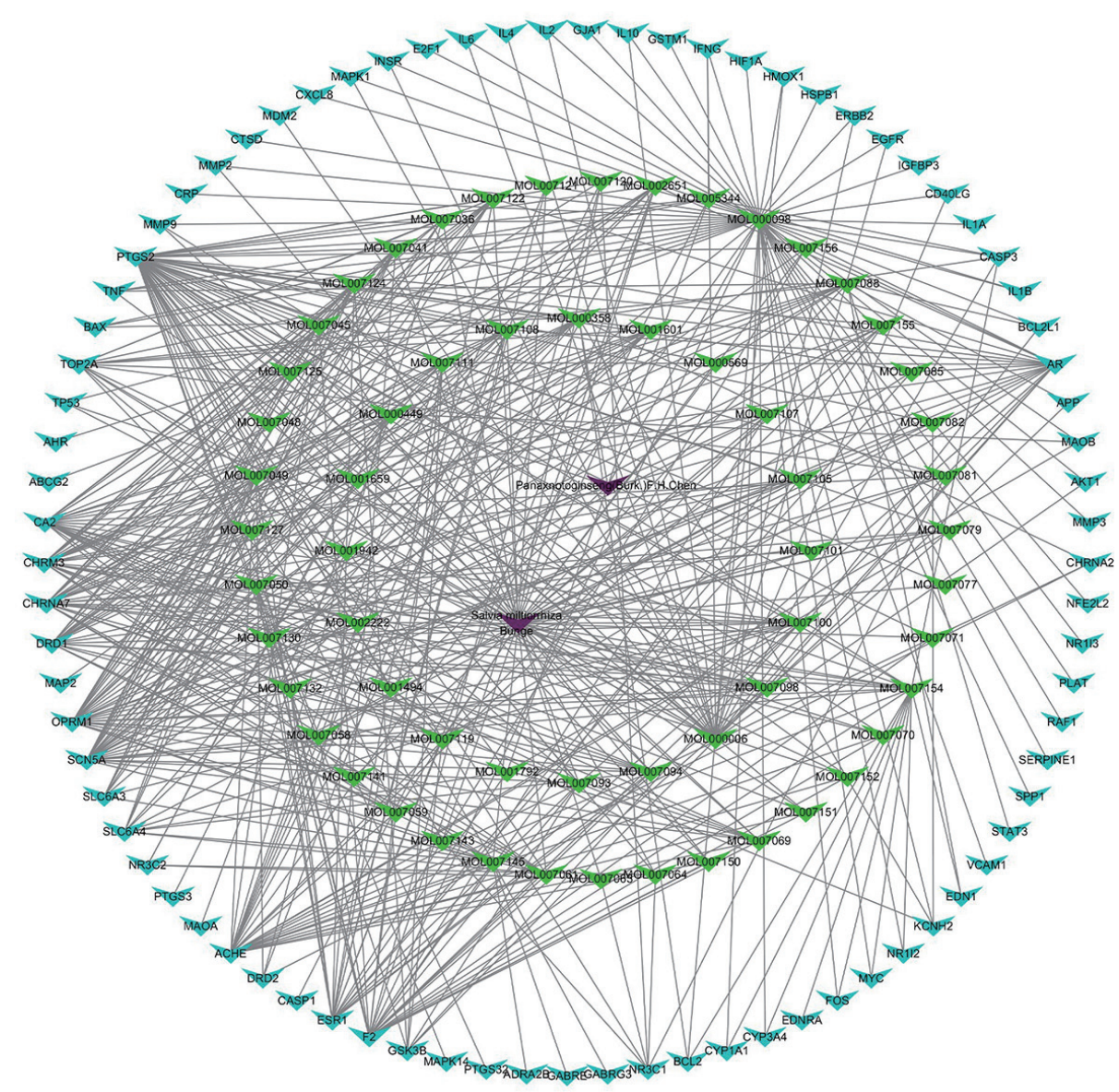

Figure 2 Mapping of Compound Danshen Dropping Pills (CDDP)- and epilepsy-related targets, 79 common targets were showed. Purple node denoted for drug, green nodes denoted for bioactive components, and blue nodes denoted for predicted targets.

less than 2, indicating that the important active ingredients of CDDP combined well with the hub targets. This indicates that the results of this study are reliable, as shown in Figure 10.

\section{Discussion}

Epilepsy is a very common neurological disease, characterized by recurrent epileptic seizures or abnormal behavior, feeling, and even loss of consciousness caused by the abnormal discharge of brain neurons (23). The annual incidence rate of active epilepsy in China is about 3.79 per thousand (24). The prevalence is the highest in 1-year old, and gradually decreases with age. Relevant studies $(25,26)$ have shown that about a quarter of epilepsy cases can be prevented. In patients with epilepsy, about $60-70 \%$ of patients can achieve zero seizure through antiepileptic drugs, but the long-term use of antiepileptic drugs may result in some side effects. In addition, a considerable number of epileptic patients are still unable to control seizures through drugs or surgery (27). Based on the above situation, the current research on epilepsy is mainly divided into two aspects: (I) the prevention and treatment of epilepsy, and (II) the health monitoring and nursing of patients with epilepsy. These studies involve multiple fields, including biology, medicine, rehabilitation, electronics, and computer science.

In recent years, research into the mechanism of traditional Chinese medicine epilepsy treatment has increased. Numerous studies have shown that traditional Chinese medicine can inhibit the occurrence and development of epilepsy by inhibiting apoptosis, regulating oxidative stress, and inhibiting inflammatory reactions $(28,29)$. Epilepsy has the characteristics of long course 
A

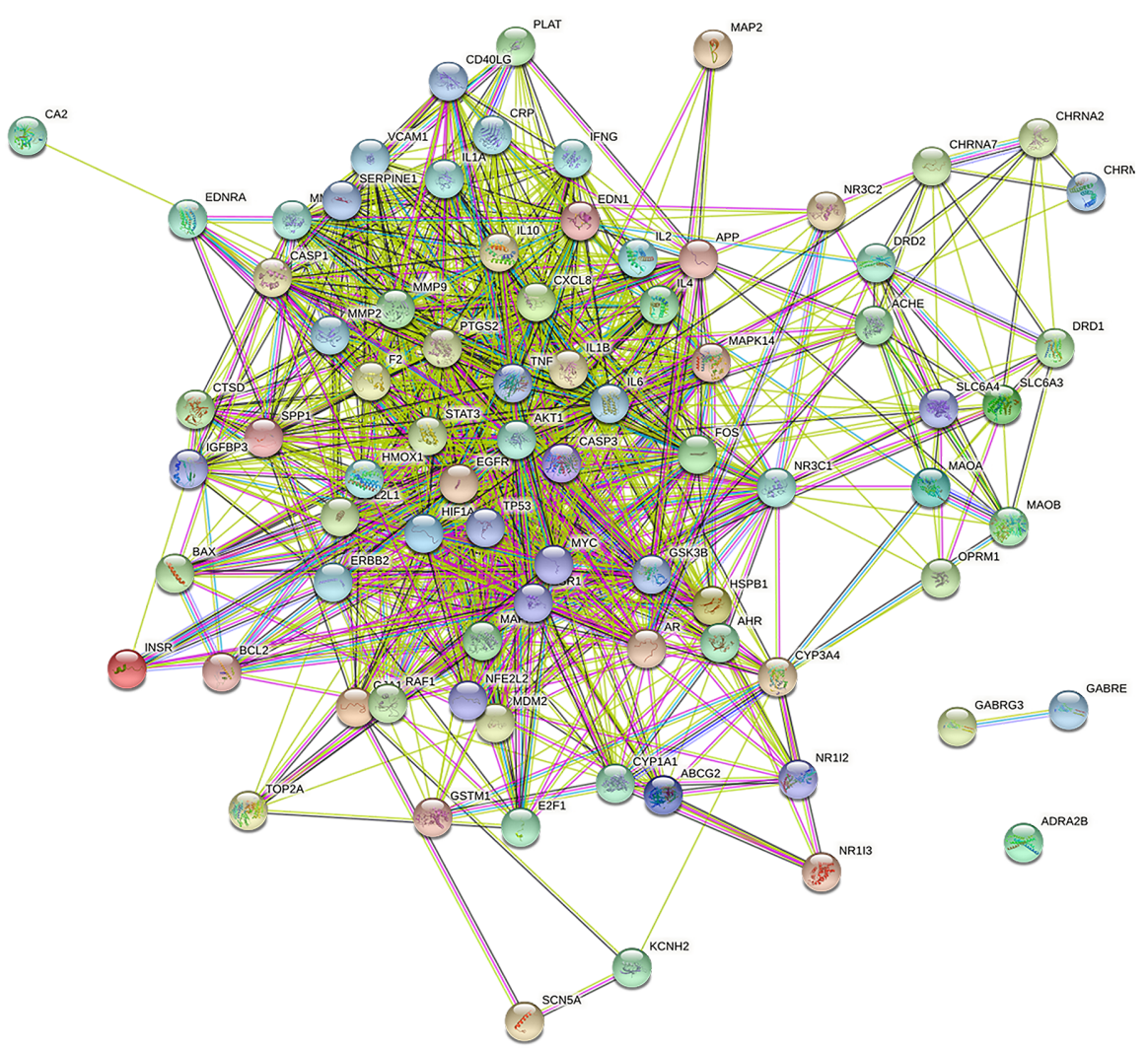

B

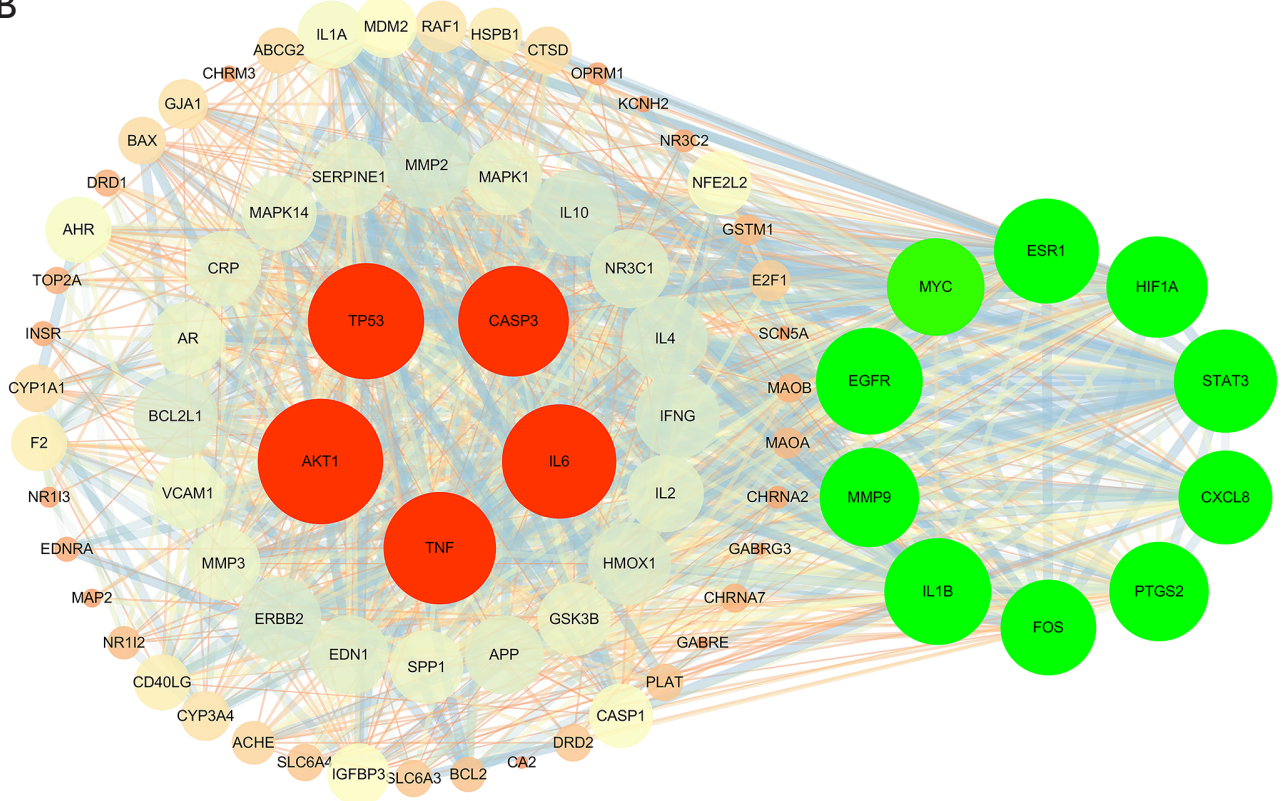

Figure 3 Common-target network. (A) Protein-protein interaction mapping by string database; (B) interaction network of Compound Danshen Dropping Pills (CDDP)-related targets. The red nodes represented the key targets. 
A

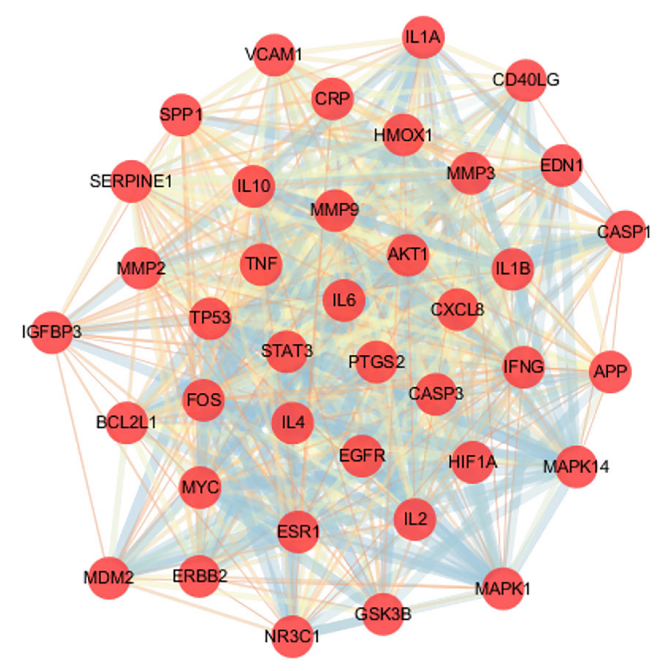

B

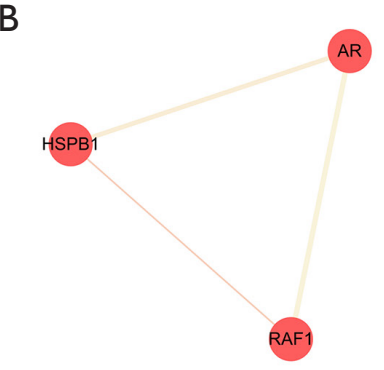

D

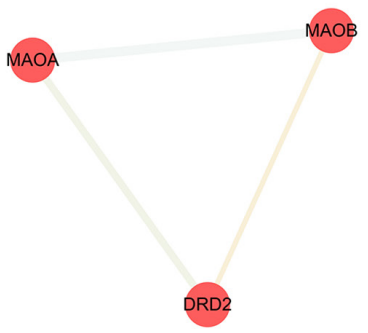

C

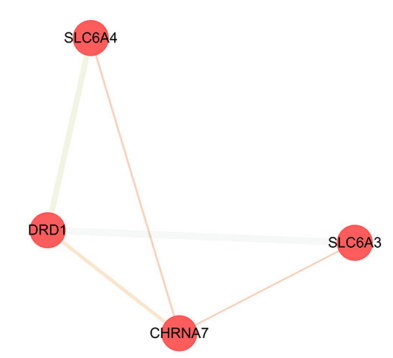

$\mathrm{E}$

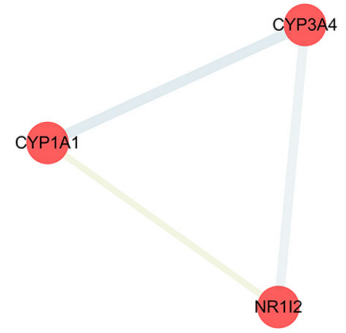

Figure 4 Subnetwork graph of protein-protein interaction. (A) Score =31.737; (B) Score =3.33; (C) Score =3; (D) Score =3; (E) Score =3.
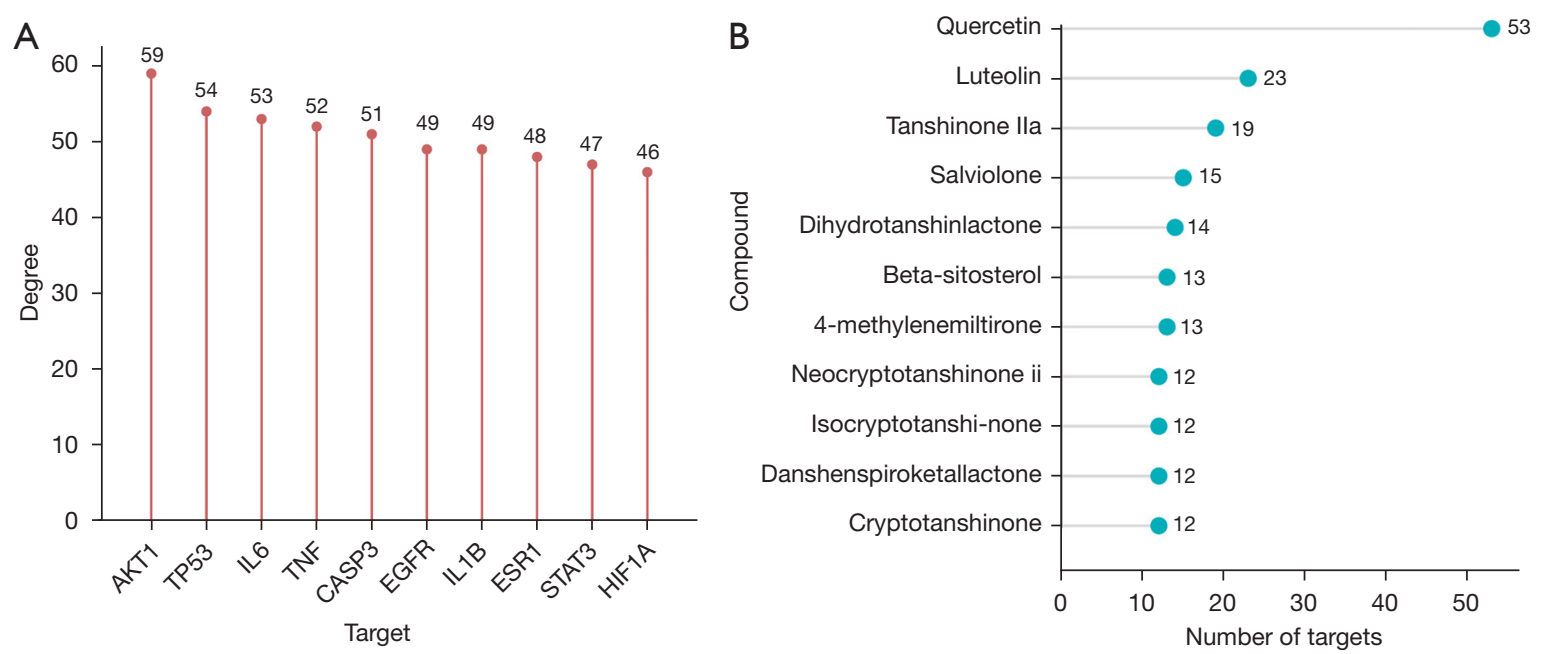

Figure 5 Hub targets and active ingredients. (A) Degree level of each hub-target; (B) number of targets corresponding to important active ingredients.

of disease, recurrent seizures, and curative difficulty. Traditional Chinese medicine has the characteristics of obvious curative effect, multi-target, multi-channel, as well as minimal toxicity and side effects in the prevention and treatment of epilepsy. Therefore, in-depth study of the signaling pathway mechanisms in epilepsy and the development of more effective antiepileptic drugs are the focus of future research.

Based on the method of network pharmacology, we clarify the mechanism of CDDP in the treatment of epilepsy. Quercetin is one of the most abundant flavonoids in nature, and has antioxidant, anti-inflammatory, antitumor, and antiviral neuroprotective effects $(30,31)$. Quercetin can reduce lipid peroxidation and increase endogenous antioxidant enzymes in the brain, so as to reduce the frequency and severity of seizures (32). It is reported that quercetin can resist apoptosis and protect neurons by increasing $\mathrm{X}$-linked inhibitor of apoptosis protein 


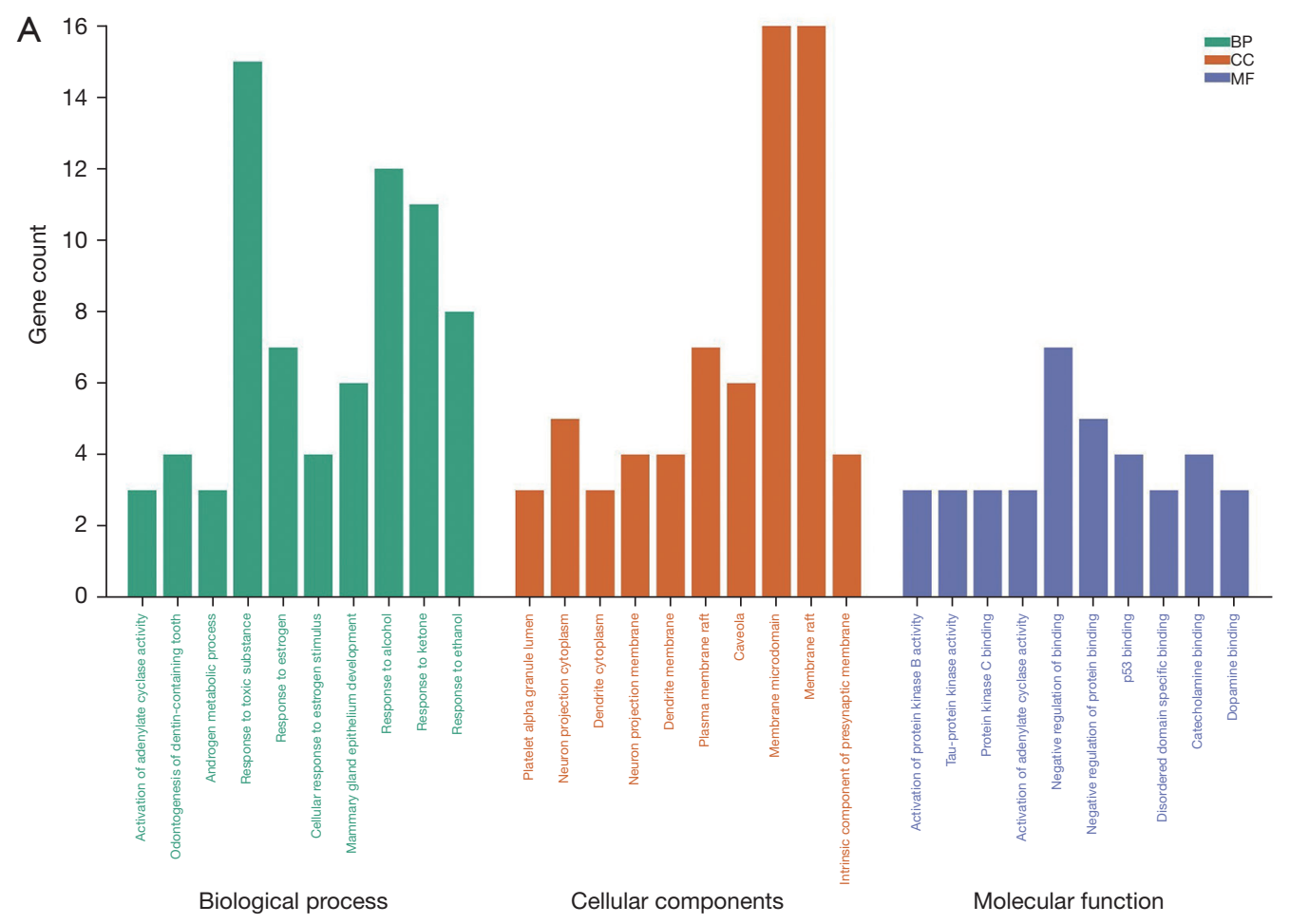

B

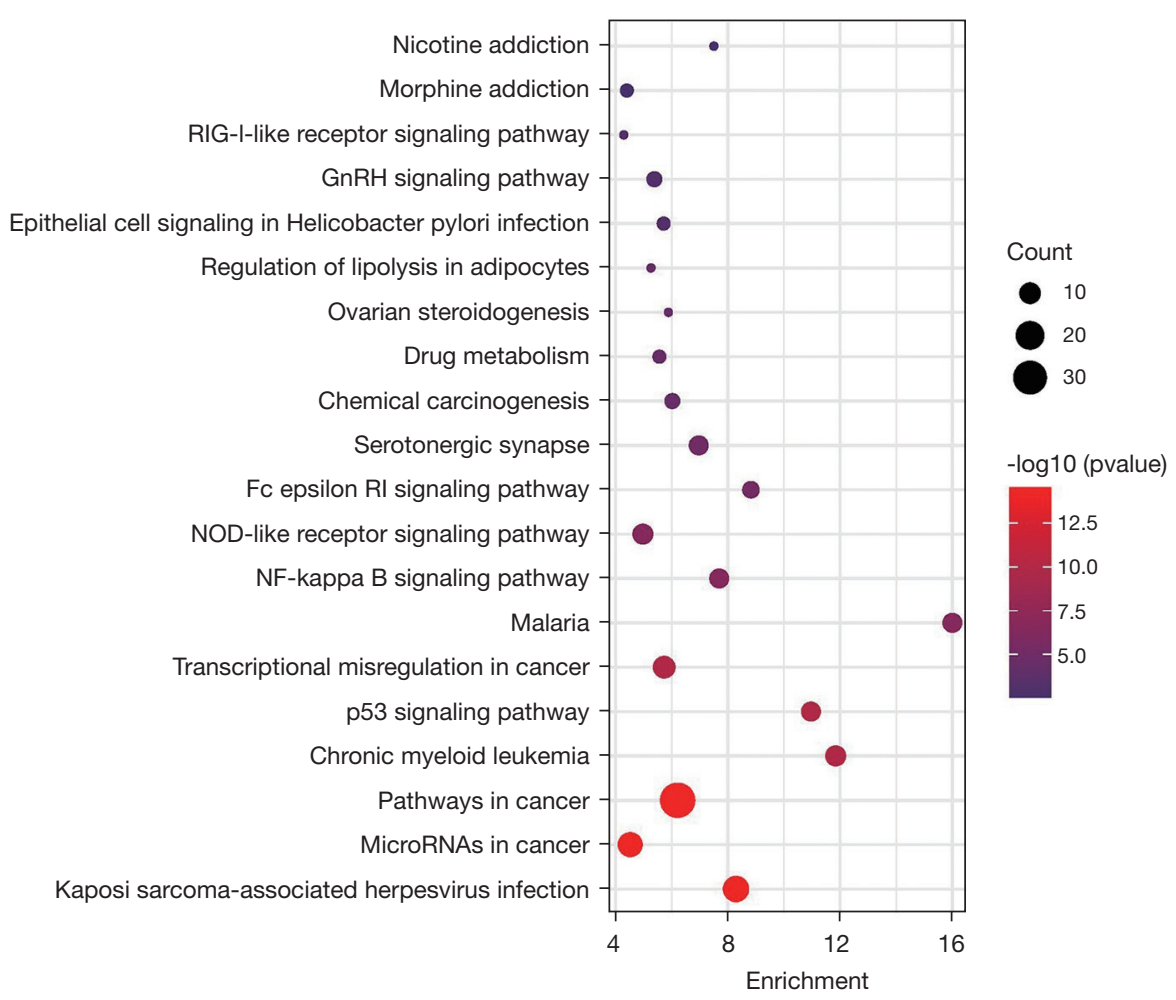

Figure 6 Enrichment of gene ontology (GO) and Kyoto Encyclopedia of Genes and Genomes (KEGG) pathways of Compound Danshen Dropping Pills (CDDP) treating epilepsy. (A) Enriched gene ontology terms; (B) enriched KEGG pathways. 
Table 2 Basic information of the key targets

\begin{tabular}{lccc}
\hline GC ID & Symbol & Degree & Description \\
\hline GC14M104769 & AKT1 & 59 & AKT serine/threonine kinase 1 \\
GC17M007661 & TP53 & 54 & Tumor protein P53 \\
GC07P022725 & IL6 & 53 & Interleukin 6 \\
\hline
\end{tabular}

A

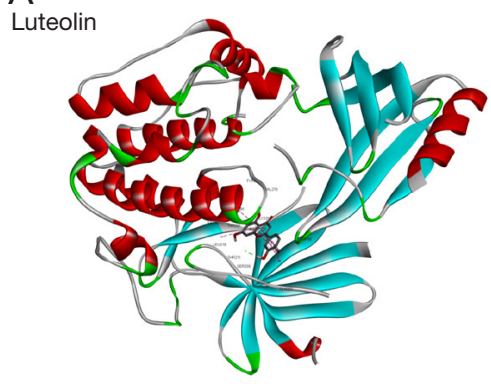

B

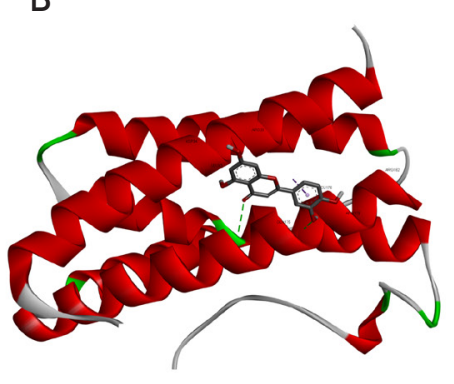

C

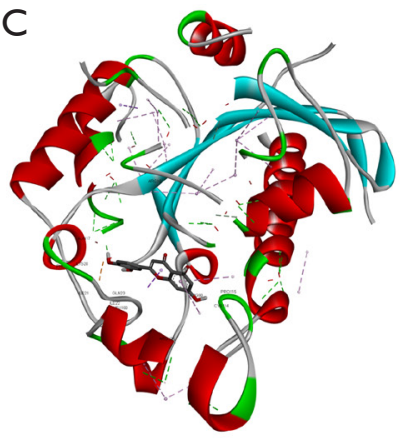

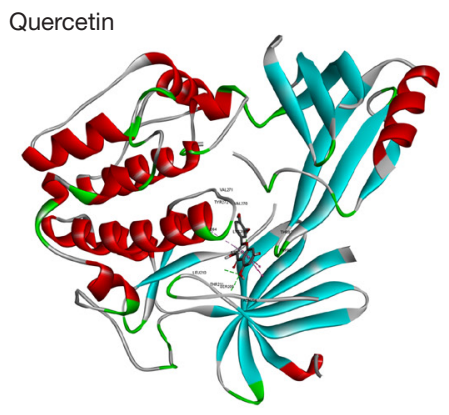
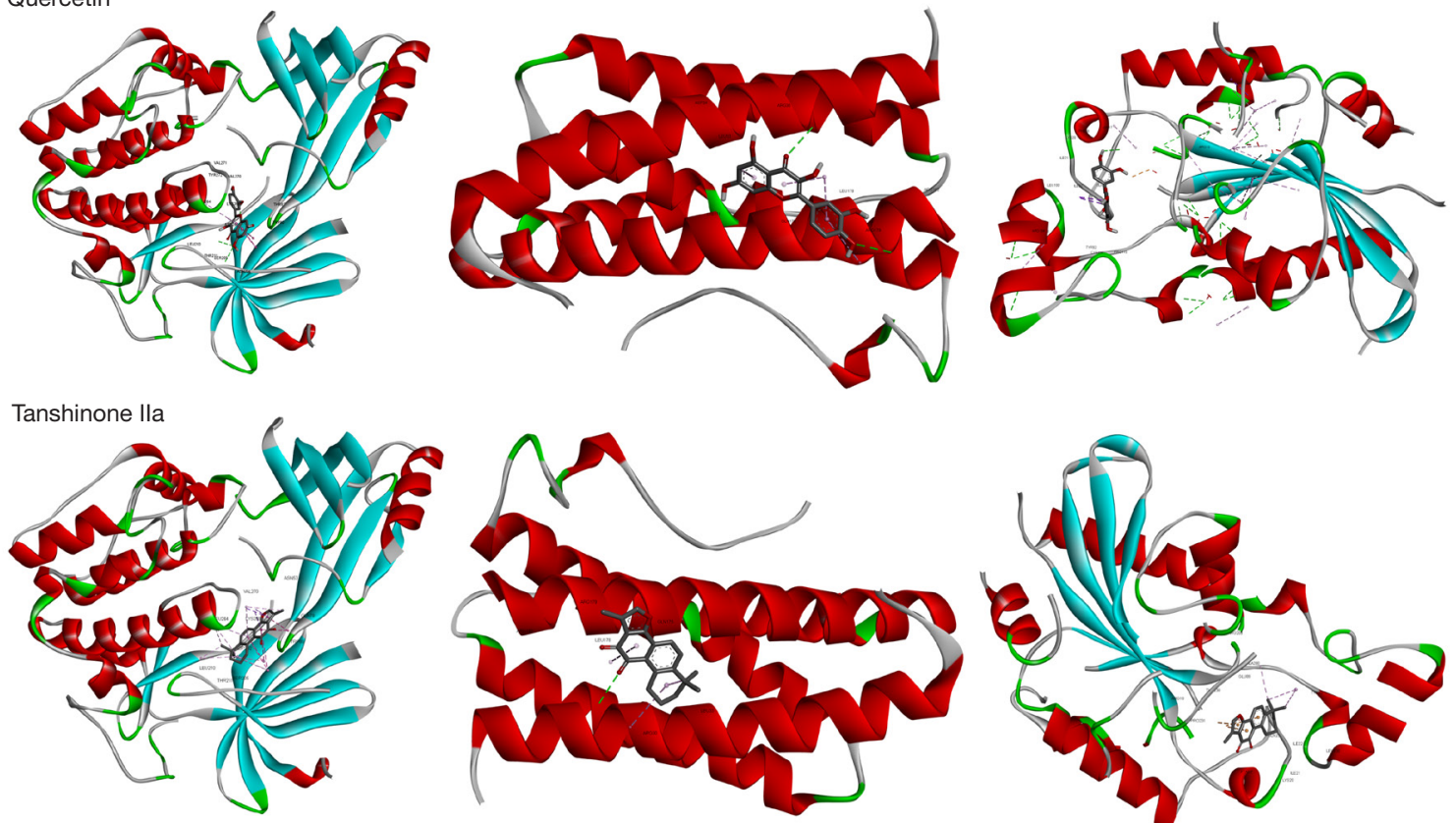

AKT1

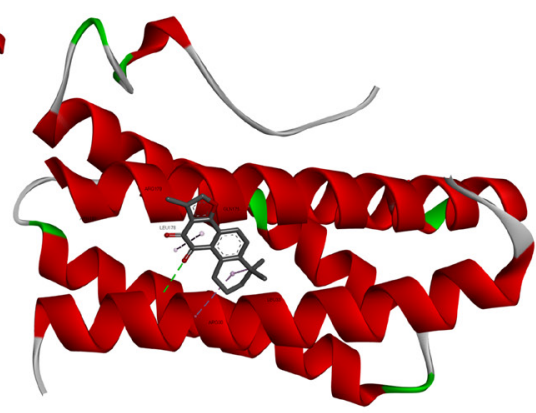

IL6

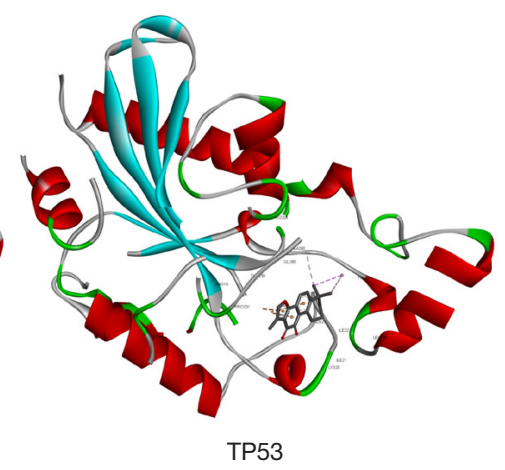

Figure 7 The three targets were as follows: interleukin-6 (IL6, PDB ID: 1alu), AKT serine/threonine kinase 1 (AKT1, PDB ID: 6s9x), and tumor protein p53 (TP53, PDB ID: 3dcy). The top three compounds were luteolin, quercetin, and tanshinone IIa. The binding energy ranged from -10.9 to $-5.10 \mathrm{kcal} / \mathrm{mol}$. Structural model of active ingredients with key targets. (A) Structural model of $A K T I$ with luteolin, quercetin, and tanshinone IIa; (B) structural model of IL6 with luteolin, quercetin, and tanshinone IIa; (C) structural model of TP53 with luteolin, quercetin, and tanshinone IIa. 

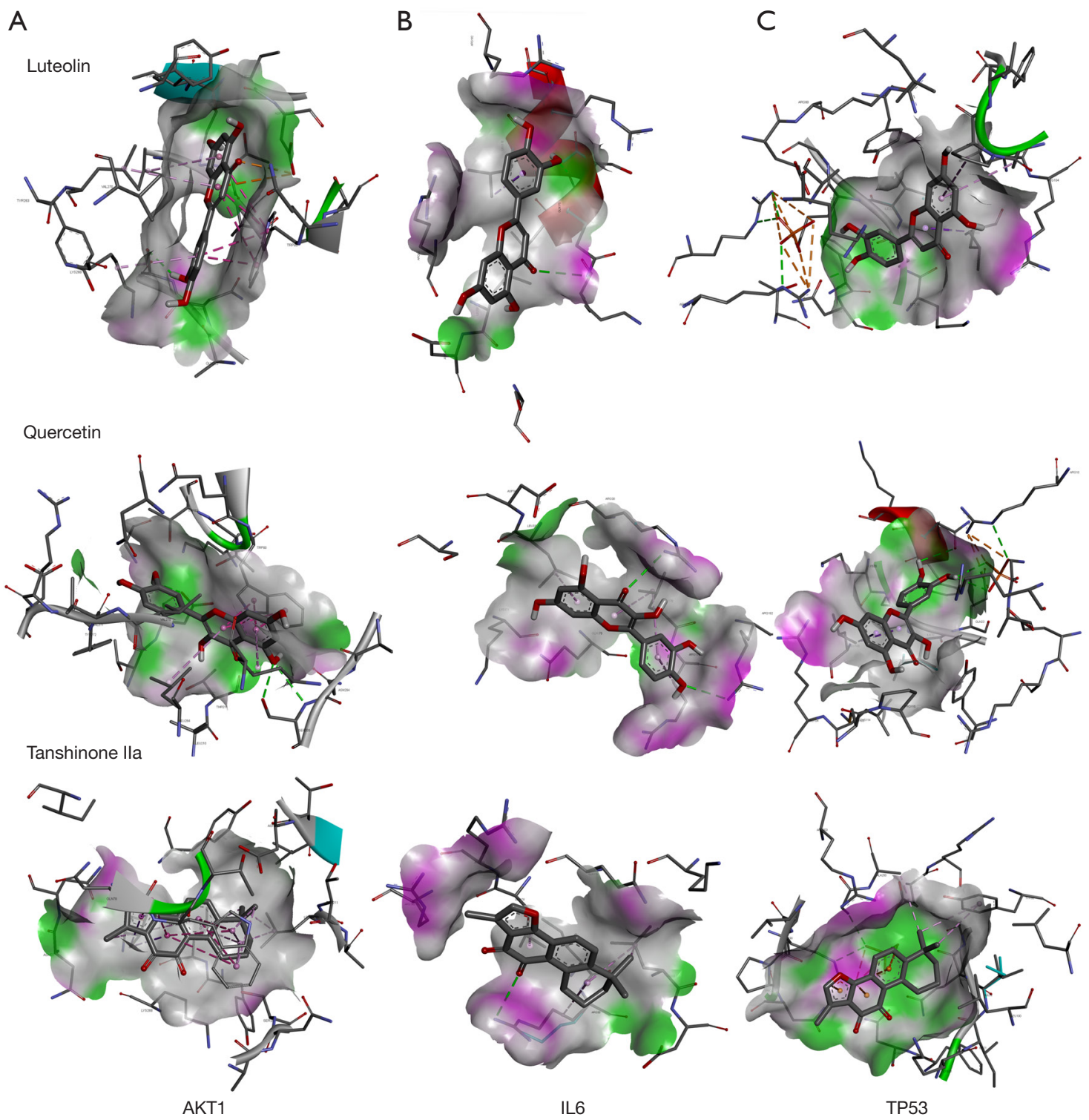

Figure 8 Binding mode of proteins and ligands. (A) binding mode of $A K T$ serine/threonine kinase 1 (AKT1) with luteolin, quercetin, and tanshinone IIa; (B) binding mode of interleukin-6 (IL6) with luteolin, quercetin, and tanshinone IIa; (C) binding mode of tumor protein $p 53$ (TP53) with luteolin, quercetin, and tanshinone IIa.

(XIAP) expression and inhibiting caspase- 3 activity in the hippocampus (33). Based on Morris water maze experiment, D'Hooge et al. found that the latency of looking for the platform was prolonged and the residence time in the hidden platform quadrant was shortened, indicating the decline in learning and memory ability (34). Tanshinone IIa is the main fat soluble component of Salvia miltiorrhiza, a diterpene quinone compound. It has pharmacological activities in cardiovascular diseases, anti-tumor, anti-inflammatory, anticoagulant, and improving organ fibrosis (35). Using pentylenetetrazole induced juvenile zebrafish and mouse epilepsy models, it was found that tanshinone IIa may play an anticonvulsant role by activating the $\gamma$-aminobutyric acid $(G A B A)$ signaling pathway. Tanshinone IIa can also protect against lead-induced neurobehavioral defects in rats (36). In addition, our research group's previous study found that tanshinone IIa may alleviate epileptic seizures in epileptic rats by regulating the brain-derived neurotrophic 

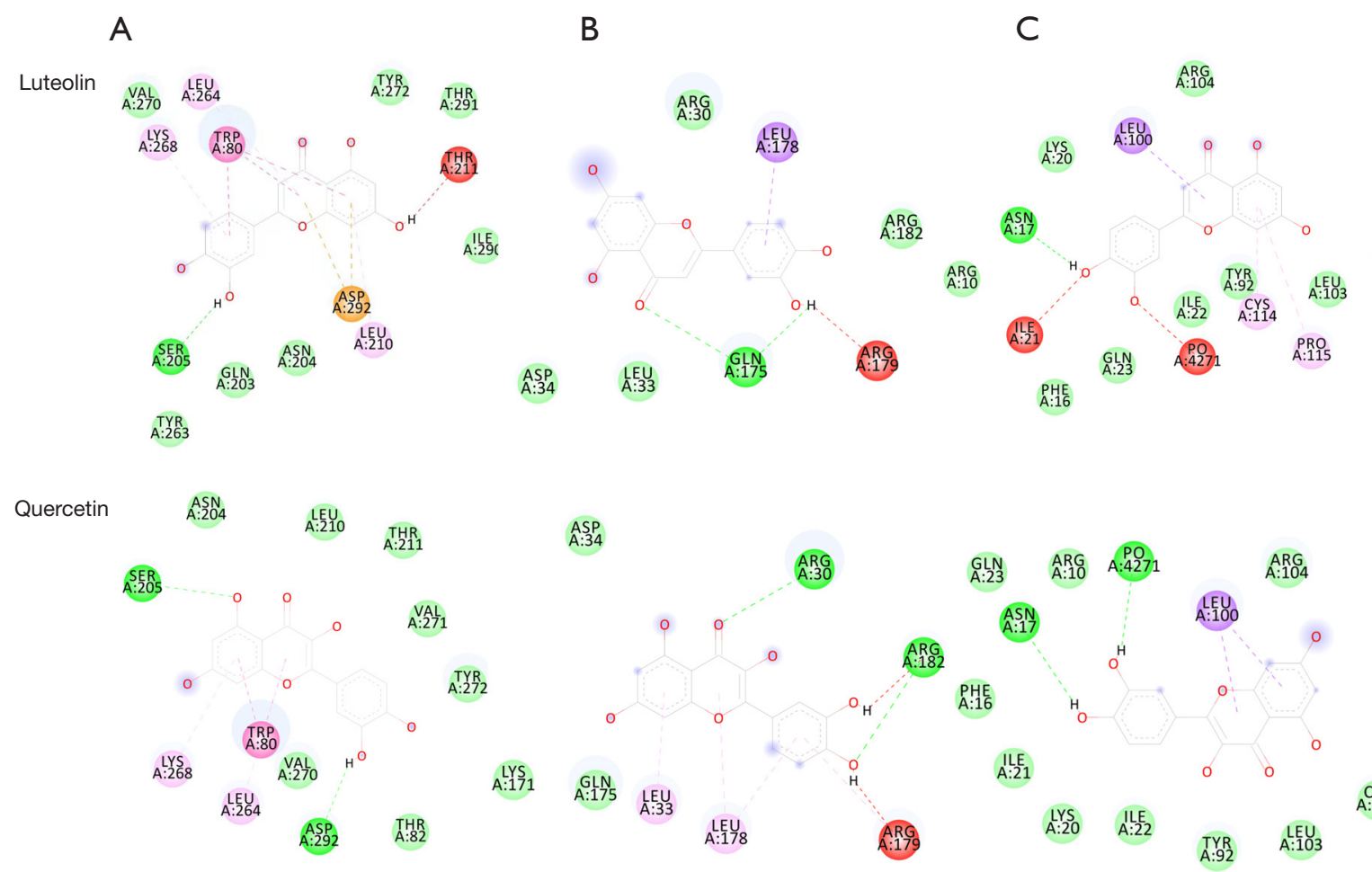

A.sp
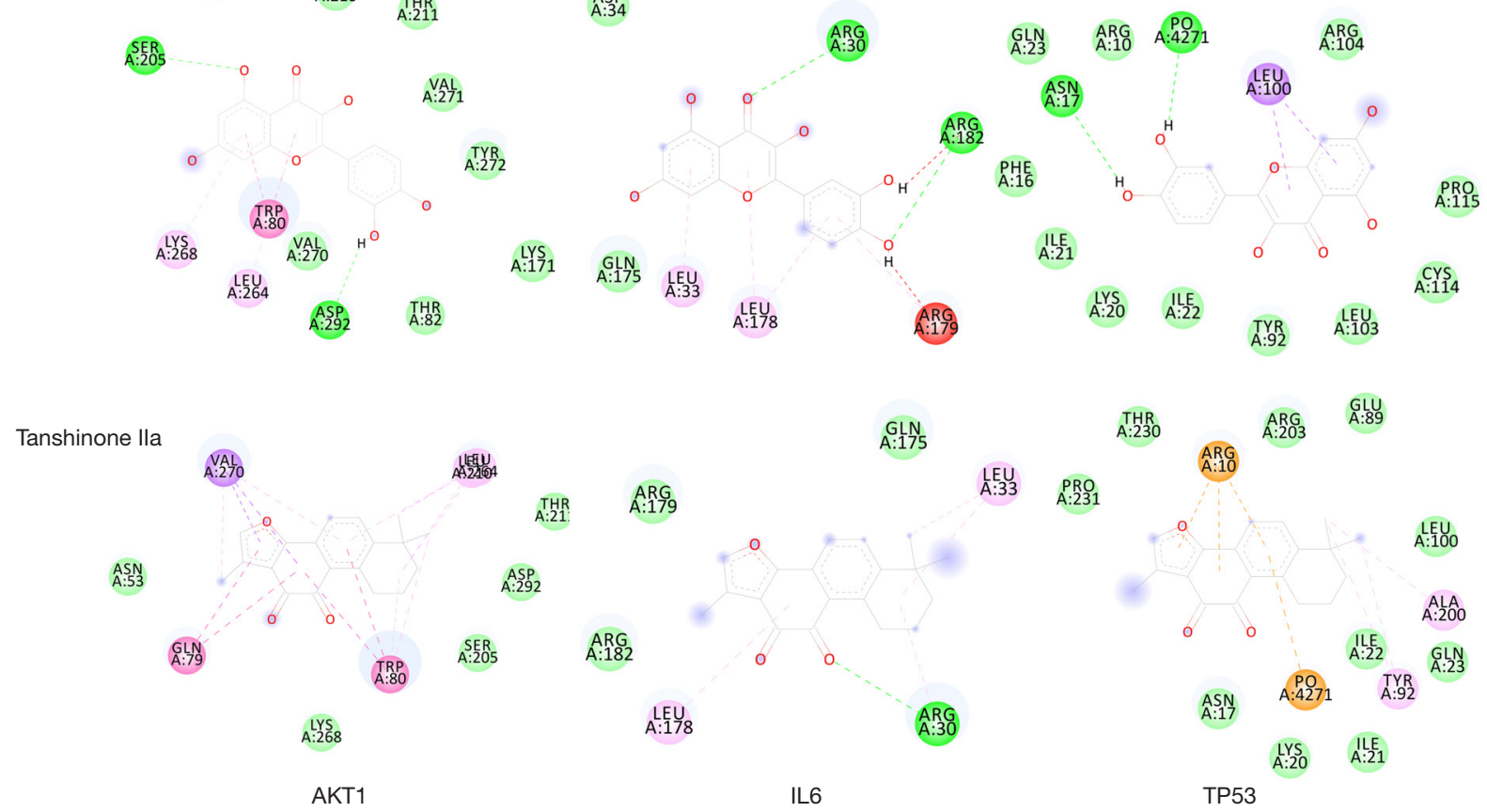

Figure 9 Binding site of key targets and active ingredients. (A) binding site of $A K T$ serine/threonine kinase 1 (AKT1) with luteolin, quercetin, and tanshinone IIa; (B) binding site of interleukin-6 (ILG) with luteolin, quercetin, and tanshinone IIa; (C) binding site of tumor protein $p 53$ (TP53) with luteolin, quercetin, and tanshinone IIa.

factor- Tyrosine Kinase receptor B (BDNF TrkB) pathway, enhance the expression of hippocampal synaptic remodeling proteins, syn and PSD-95, in epileptic rats, regulate synaptic plasticity, and subsequently improve the cognitive function of epileptic rats.

Interleukin 6 (IL6) is a commonly used inflammatory factor in the clinic, which can promote the proliferation and functional changes of glial cells and participate in the regulation of neuronal function. Studies have shown that IL6 is a neuroprotective factor, which can protect nerve cells, repair damaged neurons and reduce epileptic brain injury $(37,38) . P 38$ is an important member of the mitogenactivated protein kinase $(M A P K)$ family. It causes nuclear translocation after activation and leads to phosphorylation activation for many protein kinases and transcription factors, and it is very important for apoptosis (39). P53 protein is the downstream transcription factor of $\mathrm{p} 38$, and mainly acts to cause programmed apoptosis, and negatively regulate cell division and proliferation. It has been found that up-regulation of $p 53$ expression can induce tumor cell 
Table 3 Free binding energy and RMSD of the key active components of CDDP and key targets

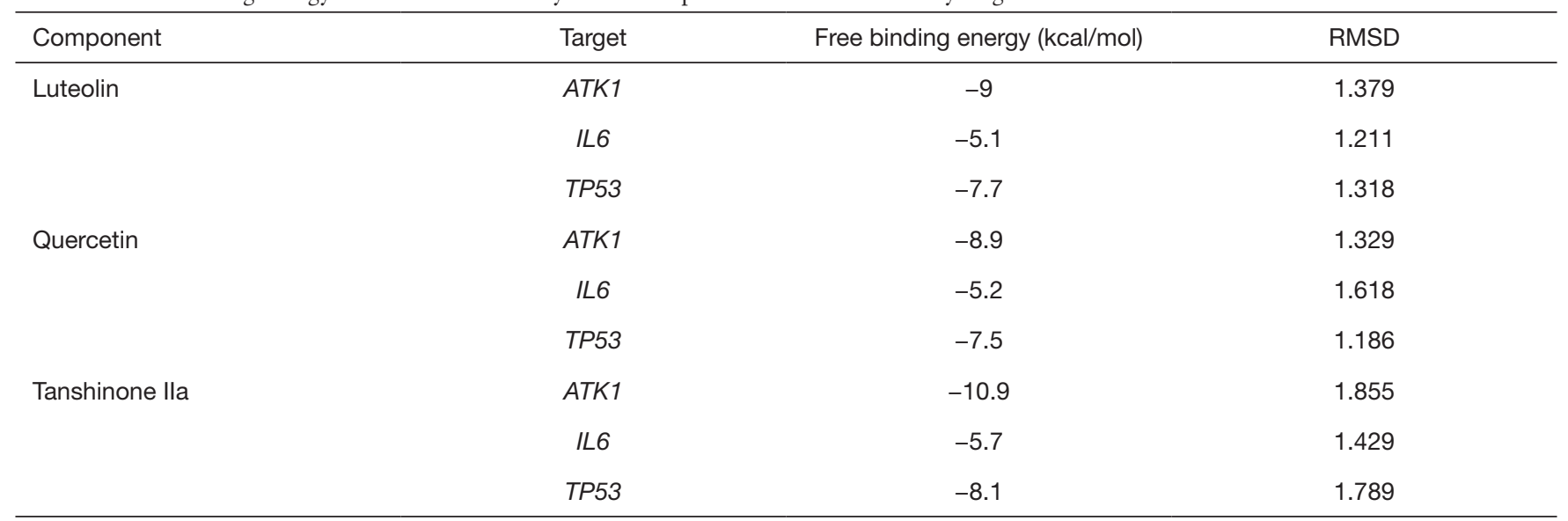

RMSD, Root Mean Square Deviation; CDDP, Compound Danshen Dropping Pills; AKT1, AKT serine/threonine kinase 1; TP53, tumor protein P53; IL6, interleukin 6.

A

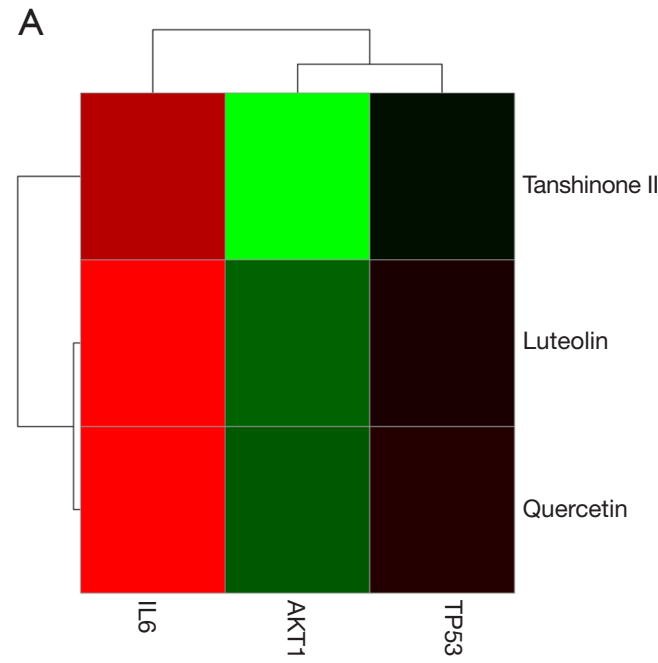

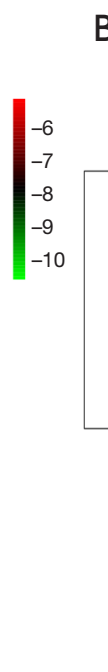
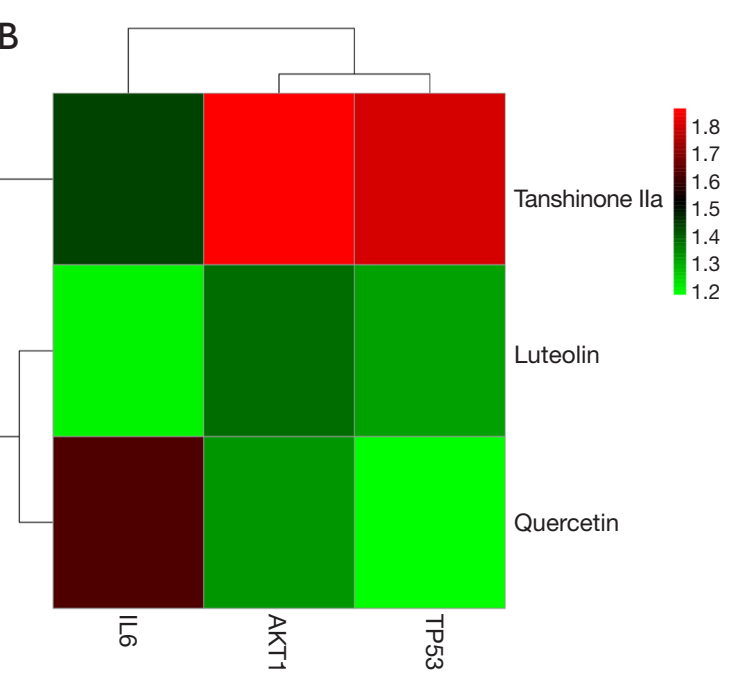

Figure 10 Heat map of binding energy (A) and Root Mean Square Deviation (RMSD) (B).

apoptosis, and inhibiting the $038 / 1553$ pathway can protect the apoptosis of ischemic stroke neurons (40). It was found that many apoptotic neuronal pyramidal cells gather in hippocampal CA1 area after epilepsy, and the expression of the $p 53$ protein increased in pyramidal cells. However, CDDP also have some limitations. Individual patients will have skin rash, dyspnea, decreased blood pressure, and even shock. Long term use may cause stomach pain, indigestion, loss of appetite and other symptoms.

\section{Conclusions}

Our research is the first to investigate the underlying mechanism of cisplatin therapy for epilepsy using webbased pharmacological analysis. The results showed that the multiple pathways, targets, and components of CDDP had synergistic effects in the treatment of epilepsy. Our study can conduct deeper studies upon mechanisms of traditional Chinese medicine treatments for epilepsy, and provides a glimpse into for studying potential mechanisms of TCM treatments in other forms of mental illness using network pharmacology methods. Our study provides a mechanism for CDDP treatment of epilepsy based on the existing database. To guarantee quality and rationality of results, advanced exploratory confirmation in vivo and in vitro is necessary. 


\section{Acknowledgments}

Funding: The study was supported by Hainan Province Key R\&D Program (Grant No. ZDYF2020165), and Natural Science Foundation of Hainan Province (Grant No. 820MS134).

\section{Footnote}

Reporting Checklist: The authors have completed the STREGA reporting checklist. Available at https://atm. amegroups.com/article/view/10.21037/atm-22-195/rc

Conflicts of Interest: All authors have completed the ICMJE uniform disclosure form (available at https://atm. amegroups.com/article/view/10.21037/atm-22-195/coif). The authors have no conflicts of interest to declare.

Ethical Statement: The authors are accountable for all aspects of the work in ensuring that questions related to the accuracy or integrity of any part of the work are appropriately investigated and resolved. The study was conducted in accordance with the Declaration of Helsinki (as revised in 2013).

Open Access Statement: This is an Open Access article distributed in accordance with the Creative Commons Attribution-NonCommercial-NoDerivs 4.0 International License (CC BY-NC-ND 4.0), which permits the noncommercial replication and distribution of the article with the strict proviso that no changes or edits are made and the original work is properly cited (including links to both the formal publication through the relevant DOI and the license). See: https://creativecommons.org/licenses/by-nc-nd/4.0/.

\section{References}

1. Kola PK, Akula A, Nissankara Rao LS, et al. Naringin in a combined therapy with phenytoin on pentylenetetrazoleinduced kindling in rats. Epilepsy Behav 2018;89:159-68.

2. Thijs RD, Surges R, O'Brien TJ, Sander JW. Epilepsy in Adults[J]. The Lancet. 2019;393:689-701.

3. Al-Otaibi F. An overview of structurally diversified anticonvulsant agents. Acta Pharm 2019;69:321-44.

4. Bauer PR, de Goede AA, Stern WM, et al. Longinterval intracortical inhibition as biomarker for epilepsy: a transcranial magnetic stimulation study. Brain 2018;141:409-21.
5. GBD 2015 Disease and Injury Incidence and Prevalence Collaborators. Global, regional, and national incidence, prevalence, and years lived with disability for 310 diseases and injuries, 1990-2015: a systematic analysis for the Global Burden of Disease Study 2015. Lancet 2016;388:1545-602.

6. MacDonald SC, Bateman BT, McElrath TF, et al. Mortality and Morbidity During Delivery Hospitalization Among Pregnant Women With Epilepsy in the United States. JAMA Neurol 2015;72:981-8.

7. Vu LC, Piccenna L, Kwan P, et al. New-onset epilepsy in the elderly. Br J Clin Pharmacol 2018;84:2208-17.

8. Seid S, Demilew D, Yimer S, et al. Prevalence and Associated Factors of Mental Distress among Caregivers of Patients with Epilepsy in Ethiopia: A Cross-Sectional Study Design. Psychiatry J 2018;2018:2819643.

9. Keller AE, Whitney R, Li SA, et al. Incidence of sudden unexpected death in epilepsy in children is similar to adults. Neurology 2018;91:e107-11.

10. Kawakami Y, Fujii S, Ishikawa G, et al. Valproate-Induced Polycystic Ovary Syndrome in a Girl with Epilepsy: A Case Study. J Nippon Med Sch 2018;85:287-90.

11. Zaccara G, Giorgi FS, Amantini A, et al. Why we prefer levetiracetam over phenytoin for treatment of status epilepticus. Acta Neurol Scand 2018;137:618-22.

12. Nakamura K, Ohbe H, Matsui H, et al. Phenytoin versus fosphenytoin for second-line treatment of status epilepticus: propensity score matching analysis using a nationwide inpatient database. Seizure 2020;80:124-30.

13. Ma C, Xu T, Sun X, et al. Network Pharmacology and Bioinformatics Approach Reveals the Therapeutic Mechanism of Action of Baicalein in Hepatocellular Carcinoma. Evid Based Complement Alternat Med 2019;2019:7518374.

14. Dong Y, Tao B, Xue X, et al. Molecular mechanism of Epicedium treatment for depression based on network pharmacology and molecular docking technology. BMC Complement Med Ther 2021;21:222.

15. Xinqiang S, Yu Z, Ningning Y, et al. Molecular mechanism of celastrol in the treatment of systemic lupus erythematosus based on network pharmacology and molecular docking technology. Life Sci 2020;240:117063.

16. Jiang Y, Zhong M, Long F, et al. Network PharmacologyBased Prediction of Active Ingredients and Mechanisms of Lamiophlomis rotata (Benth.) Kudo Against Rheumatoid Arthritis. Front Pharmacol 2019;10:1435.

17. Deng P, Liang H, Xie K, et al. Study on the molecular 
mechanism of Guizhi Jia Shaoyao decoction for the treatment of knee osteoarthritis by utilizing network pharmacology and molecular docking technology. Allergol Immunopathol (Madr) 2021;49:16-30.

18. Feng C, Zhao M, Jiang L, et al. Mechanism of Modified Danggui Sini Decoction for Knee Osteoarthritis Based on Network Pharmacology and Molecular Docking. Evid Based Complement Alternat Med 2021;2021:6680637.

19. Ru J, Li P, Wang J, et al. TCMSP: a database of systems pharmacology for drug discovery from herbal medicines. J Cheminform 2014;6:13.

20. Li J, Zhao P, Li Y, et al. Systems pharmacology-based dissection of mechanisms of Chinese medicinal formula Bufei Yishen as an effective treatment for chronic obstructive pulmonary disease. Sci Rep 2015;5:15290.

21. Szklarczyk D, Gable AL, Lyon D, et al. STRING v11: protein-protein association networks with increased coverage, supporting functional discovery in genomewide experimental datasets. Nucleic Acids Res 2019;47:D607-13.

22. Bader GD, Hogue CW. An automated method for finding molecular complexes in large protein interaction networks. BMC Bioinformatics 2003;4:2.

23. Bernard C. Understanding and Predicting Epilepsy Life Sciences. IEEE Signal Processing Magazine 2016;33:90-5.

24. Song P, Liu Y, Yu X, et al. Prevalence of epilepsy in China between 1990 and 2015: A systematic review and metaanalysis. J Glob Health 2017;7:020706.

25. Naik GS, Kodagali R, Mathew BS, et al. Therapeutic Drug Monitoring of Levetiracetam and Lamotrigine: Is There a Need? Ther Drug Monit 2015;37:437-44.

26. Nearing K, Madhavan D, Devinsky O. Temporal lobe epilepsy: a progressive disorder? Rev Neurol Dis 2007;4:122-7.

27. Fisher R, Salanova V, Witt T, et al. Electrical stimulation of the anterior nucleus of thalamus for treatment of refractory epilepsy. Epilepsia 2010;51:899-908.

28. Schiavone S, Morgese MG, Tucci P, et al. The Therapeutic Potential of Celastrol in Central Nervous System Disorders: Highlights from In Vitro and In Vivo Approaches. Molecules 2021;26:4700.

29. Dang J, Paudel YN, Yang X, et al. Schaftoside Suppresses Pentylenetetrazol-Induced Seizures in Zebrafish via Suppressing Apoptosis, Modulating Inflammation, and Oxidative Stress. ACS Chem Neurosci 2021;12:2542-52.

30. Dajas F. Life or death: neuroprotective and anticancer effects of quercetin. J Ethnopharmacol 2012;143:383-96.

31. Dajas F, Andrés AC, Florencia A, et al. Neuroprotective actions of flavones and flavonols: mechanisms and relationship to flavonoid structural features. Cent Nerv Syst Agents Med Chem 2013;13:30-5.

32. Shuyu LI, Bo Xiao, Fangfang Bi, et al. The expression of XIAP mRNA and protein in the rat hippocampus and quercetin treatment following status epilepticus. Chinese Journal of Nervous and Mental Diseases 2008;34:542-5.

33. Brandeis R, Brandys $\mathrm{Y}$, Yehuda $\mathrm{S}$. The use of the Morris Water Maze in the study of memory and learning. Int J Neurosci 1989;48:29-69.

34. D'Hooge R, De Deyn PP. Applications of the Morris water maze in the study of learning and memory. Brain Res Brain Res Rev 2001;36:60-90.

35. Halder S, Kar R, Galav V, et al. Cadmium exposure during lactation causes learning and memory-impairment in F1 generation mice: amelioration by quercetin. Drug Chem Toxicol 2016;39:272-8.

36. Tang LM, Wang LX, Wang ZY, et al. Tanshinone IIA ameliorates lead $(\mathrm{Pb})$-induced cognitive deficits and oxidative stress in a rat pup model. Bratisl Lek Listy 2017;118:196-201.

37. Mzezewa R, Lotila J, Kiiski H, et al. A kainic acid-induced seizure model in human pluripotent stem cell-derived cortical neurons for studying the role of IL-6 in the functional activity. Stem Cell Res 2022;60:102665.

38. Huang XY, Hu QP, Shi HY, et al. Everolimus inhibits $\mathrm{PI} 3 \mathrm{~K} / \mathrm{Akt} / \mathrm{mTOR}$ and NF-kB/IL-6 signaling and protects seizure-induced brain injury in rats. J Chem Neuroanat 2021;114:101960.

39. Shi S, Cui Q, Xu J, et al. Baicalin Suppresses BilirubinInduced Apoptosis and Inflammation by Regulating p38 Mitogen-Activated Protein Kinases (MAPK) Signaling in Neonatal Neurons. Med Sci Monit 2020;26:e926441.

40. Li XK, Li Q, Xiao W,et al. Involvement of p38-p53 signal pathway in neuroprotective effects of DGMI on SH-SY5Y cells damaged by oxygen-glucose deprivation. Chinese Pharmacological Bulletin 2016;32:1699-704.

(English Language Editor: A. Kassem)

Cite this article as: Huang $\mathrm{D}$, Wen $\mathrm{X}$, Lu C, Zhang B, Fu Z, Huang Y, Niu K, Yang F. Investigating the molecular mechanism of Compound Danshen Dropping Pills for the treatment of epilepsy by utilizing network pharmacology and molecular docking technology. Ann Transl Med 2022;10(4):216. doi: 10.21037/atm-22-195 